\title{
Photoelectric and Photorefractive Properties of Polyvinylcarbazole Composites with Graphene in the Visible Spectral Range
}

\author{
A. D. Grishina ${ }^{a}$, T. V. Krivenko ${ }^{a}$, V. V. Savel'ev ${ }^{a}$, R. W. Rychwalski ${ }^{b}$, and A. V. Vannikov ${ }^{a}$ \\ ${ }^{a}$ Frumkin Institute of Physical Chemistry and Electrochemistry, Russian Academy of Sciences, \\ Leninskii pr. 31, Moscow, 119071 Russia \\ e-mail:van@elchem.ac.ru \\ ${ }^{b}$ Department of Materials Science and Manufacturing Technology, Chalmers University of Technology, \\ SE-41296 Göteborg, Sweden
}

Received February 18, 2013; in final form, March 4, 2013

\begin{abstract}
The photoelectric and photorefractive characteristics of polyvinylcarbazole (PVK) composites with $0.15 \mathrm{wt} \%$ graphene at a wavelength of $532 \mathrm{~nm}$ have been measured. The dependence of the quantum efficiency of generation of mobile charge carriers as determined from the photocurrent is well approximated by the Onsager equation calculated accurate to $E_{0}^{3}$ for the quantum yield of thermalized electron-hole pairs of $\varphi_{0}=1$ and their initial separation radius of $r_{0}=10.9 \AA$. The long-wavelength edge of optical absorption for PVK lies at $365 \mathrm{~nm}$. Thus, the photogeneration of mobile carriers during illumination of the composite at $532 \mathrm{~nm}$ is due to photoexcitation of graphene. The measurement of the photorefractive properties has revealed that the two-beam coupling gain coefficient is $\Gamma=50 \mathrm{~cm}^{-1}$ at $E_{0}=150 \mathrm{~V} / \mu \mathrm{m}$ and equal intensities of incident beams. It has been found that at $E_{0}=83.3 \mathrm{~V} / \mu \mathrm{m}$, the two-beam coupling gain coefficient increases from 8 to $14 \mathrm{~cm}^{-1}$ as the ratio of incident beam intensities $I_{1}(0) / I_{2}(0)$ increases from 1 to 2.4 .
\end{abstract}

DOI: $10.1134 / \mathrm{S} 001814391304005 \mathrm{X}$

We consider the photoelectric and photorefractive characteristics of polyvinylcarbazole (PVK) composites with graphene measured at the 532-nm emission wavelength of a DHOM-M-532 continuous-wave solid-state laser. The optical and nonlinear optical properties of these composites and their photoelectric and photorefractive characteristics were investigated in our previous study [1] using 1064-nm laser radiation. As in the earlier studies [3-5], unplasticized layers with a glass transition temperature of abut $200^{\circ} \mathrm{C}$ were used. There is no chromophore orientation polarization in a rigid-chain polymer. Since the random centrosymmetric distribution of chromophore orientations arises as a result of layer casting, the second-order electric susceptibility is zero: $\chi^{(2)}=$ $N f^{3} \beta\left\langle\cos ^{3} \xi\right\rangle=0$ inasmuch as the mean $\left\langle\cos ^{3} \xi\right\rangle$ is zero. (Here, $\beta$ is the second-order molecular polarizability, $f=\left(n_{0}^{2}+2\right) / 3$ is the Lorentz correction factor, and $\xi$ is the angle between the chromophore principal axis and the direction of an applied electric field $E_{0}$.) Consequently, the chromophores that possess third-order nonlinearity are responsible for the photorefractive characteristics in these layers, since the third-order electric susceptibility defined by $\chi^{(3)}=N \gamma f^{4}\left\langle\cos ^{4} \xi\right\rangle$ has a nonzero value in the case of random distribution of chromophores because the mean is $\left\langle\cos ^{4} \xi\right\rangle=1 / 5$ (here, $\gamma$ is the third order-molecular polarizability).
In [1] we measured the third-order susceptibility in a PVK $/ \leq 0.15$ wt $\%$ graphene composite by the $z$-scan technique using $1030-\mathrm{nm}$ femtosecond laser radiation. It was found that the real part of the susceptibility is considerably greater than its imaginary term and the total susceptibility of the sample is determined by its real term, which is close to $\chi^{(3)}=1.8 \times 10^{-9}$ esu.

\section{EXPERIMENTAL}

Graphene was dispersed in tetrachloroethane (TCE) for $48 \mathrm{~h}$ in a UZDN-A ultrasonic disperser, as had been described in [1]. Then, the dispersion was allowed to settle, with the undertreated graphene partly precipitating as black sediment on the bottom of the vessel and partly accumulating on the surface. Next, the upper black layer was removed and the homogeneous light gray dispersion solution was withdrawn with a micropipette, to which PVK was added in an amount to have $0.15 \mathrm{wt} \%$ graphene in the solid composite after evaporation of TCE. (Because of precipitation, the amount of graphene in the composite was below $0.15 \mathrm{wt} \%$.) The mixture prepared in this way was agitated by a magnetic stirrer with heating to $60^{\circ} \mathrm{C}$. Used in [1] were the layers cast immediately after preparation of the stirred mixture. In this study, layers were cast after $\sim 6$ months of storage of the mixture; during the storage, a small quantity of the black sediment also precipitated. 


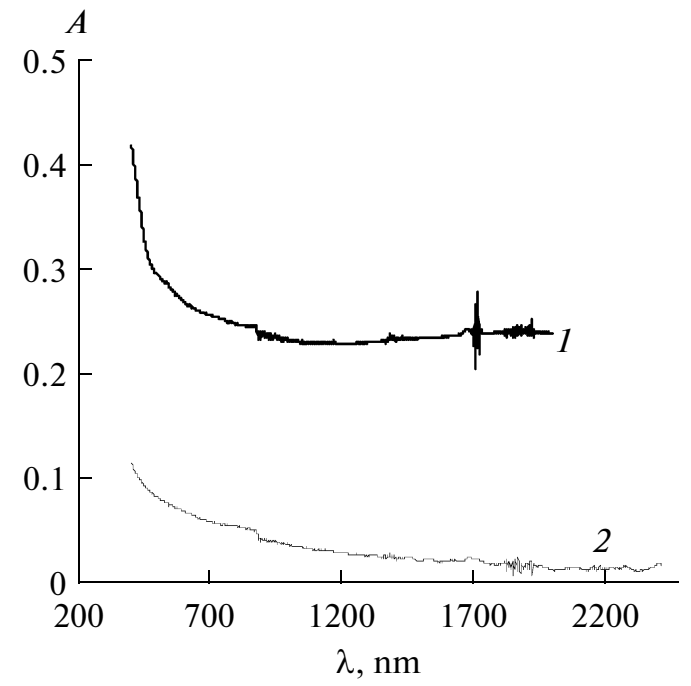

Fig. 1. Optical absorption of (1) the PVK/2.2 wt \% graphene composite cast immediately after preparation of the mixture and (2) the PVK/0.15 wt \% graphene composite cast after 6-month storage of the PVK/graphene mixture in TCE.

\section{RESULTS AND DISCUSSION}

\section{Photoelectric Characteristics}

Figure 1 shows optical spectra of graphene in a $\mathrm{PVK} / 2.2 \mathrm{wt} \%$ graphene composite cast immediately after preparation of the mixture (spectrum 1) and a $\mathrm{PVK} / \leq 0.15$ wt $\%$ graphene composite after the additional holding of the PVK/graphene mixture in TCE for about 6 months (spectrum 2). It is seen in Fig. 1 that long-wavelength optical absorption for the layer cast after the long-term storage (curve 2) is somewhat lower as compared with curve 1 measured in the sample cast immediately after preparation of the mixture. This decline is probably due to sedimentation of the most extended graphene entities responsible for the long-wavelength optical absorption. Note that after the long-term storage of the mixture, laser beam scattering by the sample completely disappeared, probably, because of the sedimentation of light-scattering extended graphene entities.

Figure 2 shows the field dependence of photocurrent and dark current in the $\mathrm{PVK} / \leq 0.15$ wt \% graphene composite as measured at $532 \mathrm{~nm}$. The layer thickness $d$ was $12 \mu \mathrm{m}$. The photocurrent was determined as the difference of the currents measured under illumination $\left(J_{\mathrm{ph}}+J_{\mathrm{d}}\right)$ and in the dark $J_{\mathrm{d}}: J_{\mathrm{ph}}=$ $\left(J_{\mathrm{ph}}+J_{\mathrm{d}}\right)-J_{\mathrm{d}}$. The field dependence of the quantum efficiency for the formation of mobile carriers was estimated from the dependence of photocurrent upon the applied electric field $J_{\mathrm{ph}}(E)$ under continuous operation of the DHOM-M-532 laser with an intensity of $I_{0}=7.32 \mathrm{~W} / \mathrm{cm}^{2}$ and was calculated by the formula:

$$
\varphi\left(E_{0}\right)=J_{\mathrm{ph}}\left(E_{0}\right) h v /\left[e I_{0}\left(1-\exp \left(-\alpha_{0} d\right)\right)\right] .
$$

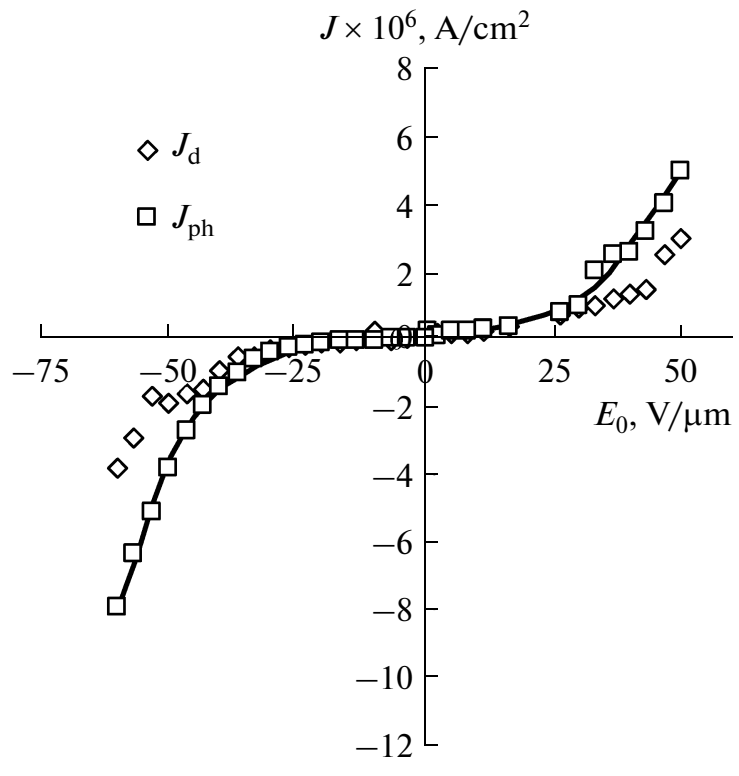

Fig. 2. Dependence of the dark current $J_{\mathrm{d}}$ and photocurrent $J_{\mathrm{ph}}$ upon the applied electric field $E_{0}$.

At $532 \mathrm{~nm}, h v=2.33 \mathrm{eV}(h v / e=2.33 \mathrm{~V})$. The quantity $\left(1-\exp \left(-\alpha_{0} d\right)\right)$ is the proportion of light energy absorbed in the layer. Figure 3 presents the quantum efficiency as a function of the applied field. The solid curve in Fig. 3 was plotted according to the Onsager equation

$$
\varphi\left(E_{0}\right)=\varphi_{0} P\left(r_{0}, E_{0}\right) .
$$

Here, $\varphi_{0}$ is the quantum yield of thermalized electron-hole pairs with an initial separation radius $r_{0}$ and $P\left(r_{0}, E_{0}\right)$ is the probability that the charges in the pairs escape recombination with the separation radius $r_{0}$. The probability was $\varphi\left(E_{0}\right)=\varphi_{0} P\left(r_{0}, E_{0}\right)$ calculated by Eq. (3) accurate to $E_{0}{ }^{3}[5,6]$ :

$$
\begin{gathered}
P\left(r_{0}, E_{0}\right)=\exp \left(-r_{\mathrm{c}} / r_{0}\right)\left\{\left[1+\left(r_{\mathrm{c}} / r_{0}\right)\left(e E_{0} r_{0} / 2 k T\right)\right.\right. \\
-\left(r_{\mathrm{c}} / r_{0}\right) K_{1}\left(e E_{0} r_{0} / 2 k T\right)^{2}+\left(r_{\mathrm{c}} / r_{0}\right) K_{2}\left(e E_{0} r_{0} / 2 k T\right)^{3},
\end{gathered}
$$

where $K_{1}=\left[2-\left(r_{\mathrm{c}} / r_{0}\right)\right] / 3, K_{2}=\left[1-\left(r_{\mathrm{c}} / r_{0}\right)+\right.$ $\left.\left(r_{\mathrm{c}} / r_{0}\right)^{2} / 6\right] / 2$, and $r_{\mathrm{c}}=190 \AA$ is the Coulomb radius.

Taking the quantum yield of thermalized electronhole pairs in Eq. (2) to be $\varphi_{0}=1$, we estimated by Eqs. (2) and (3) that the initial separation radius is $r_{0}=$ $10.9 \AA$ (curve in Fig. 3). This value is significantly higher than that of the separation radius of $r_{0}=9.8 \AA$ at $\varphi_{0}=1$, as determined at $1064 \mathrm{~nm}$ [1].

The long-wavelength edge of optical absorption by PVK lies at $365 \mathrm{~nm}$. Consequently, the photogeneration of charge carriers during illumination of the composite at both 532 and $1064 \mathrm{~nm}$ is due to photoexcitation of graphene:

Graphene $+h v \rightarrow$ Graphene*; Graphene* + $\mathrm{PVK} \rightarrow$ Graphene $^{-\cdot}+\mathrm{PVK}^{+}$and consequent hole transport $\rightarrow \mathrm{PVK}^{+}+\mathrm{PVK} \rightarrow \mathrm{PVK}+\mathrm{PVK}^{+}$etc. 
Because of the low graphene concentration of $\leq 0.15 \mathrm{wt} \%$, certain graphene "flakes" do not interact with one another and electron transport is limited.

\section{Photorefractive Properties}

When the photorefractive effect was measured, the laser beam was first split into two beams (beams 1 and 2 ), which were then brought together to intersect in the polymer layer, creating an interference pattern. The angle between the convergent beams incident on the layer was $2 \theta=15^{\circ}$. The bisector of the incident beams crossed the layer at an angle of $\phi=-45^{\circ}$. The intensity of the incident beams was $I_{1}(0)=I_{2}(0)=$ $0.073 \mathrm{~W} / \mathrm{cm}^{2}$. The measurement cell was a sandwich made from glass coated with ITO transparent conducting layer $/ \mathrm{Al}_{2} \mathrm{O}_{3}$ film/PVK-graphene composite/PVA film/ITO. Here, ITO is the $\mathrm{In}_{2} \mathrm{O}_{3}: \mathrm{SnO}_{2}$ alloy electrode onto which the dielectric $\mathrm{Al}_{2} \mathrm{O}_{3}$ film was applied by thermal deposition for reducing the dark current and the PVA (poly(vinyl alcohol)) film, which adsorbs molecular oxygen, restricted the oxygen content in the composite.

The measurements were made according to the following scheme. First, one of the beams and the electric field $E_{0}$ were successively switched on, and another beam was turned on after a while. Then, it was turned off and the field $E_{0}$ was switched off. It is well known that crossing of coherent beams causes interference and electron-hole pairs are generated in the region of bright fringes in a photosensitive polymer composite. By the action of constant electric field $E_{0}$ applied to the sample, the charges are separated and drift in opposite directions until capture in deep traps. The trapped unlike charges form a periodic spacecharge electric field $E_{\mathrm{sc}}$. The field $\left(E_{0}+E_{\mathrm{sc}}\right)$ polarizes nonlinear optical chromophores, thereby causing periodic refractive index modulation $\Delta n=$ $\left(2 \pi / n_{0}\right) \chi^{(3)}\left(E_{0}+E_{\mathrm{sc}}\right)^{2}$, i.e., providing the generation of a phase grating in the polymer layer.

Depending on the direction of the applied field $E_{0}$, the intensity of one of the beams was either amplified (object beam) or attenuated (reference beam). When a positive potential was on the output electrode (input electrode was grounded), beam 1 was amplified and beam 2 attenuated. In this case, the grating was formed on the right of interference fringes. In the case of negative potential on the output electrode, the grating was generated on the left to the interference pattern and beam 2 was amplified and beam 1 attenuated. This indicates that the capture length of photogenerated holes exceeds that of electrons. The amplification of the intensity of beam 2 upon switching beam 1 on in the case of negative potential on the output electrode, as shown in Fig. 4, reflect the formation of the grating on the left to the interference fringes. In this case, the part of light wave 1 reflected from diffraction fringes is in phase with transmitted wave 2 and has the same direction. As a result of constructive interference,

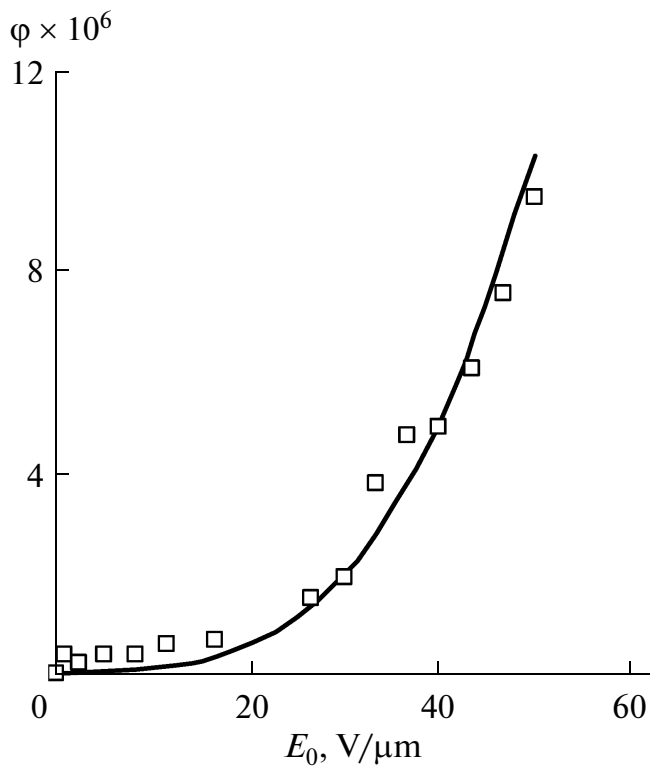

Fig. 3. Dependence of the quantum efficiency $\varphi\left(E_{0}\right)$ upon the applied electric field $E_{0}$. The symbols refer to the experimental data and the curve has been constructed according to the Onsager equation calculated accurate to $E_{0}^{3}$ for a quantum yield of thermalized electron-hole pairs of $\varphi_{0}=1$ and their initial separation radius of $r_{0}=10.9 \AA$.

beam 2 is amplified. The reflected portion of beam 2 also coincides with beam 1 by direction, but it is in antiphase, thus resulting in their destructive interference and a reduction in intensity of beam 1 . Note that the grating period $\Lambda=\lambda /\left(2 n_{0} \sin \theta\right)$ is $1359 \mathrm{~nm}$ at $\lambda=$ $532 \mathrm{~nm}, n_{0}=1.5$, and $\theta=7.5^{\circ}$. The diameter of the crossing region of laser beams was about $4 \mathrm{~mm}$.

The amplification kinetic curves of beam 2 in Fig. 4 are well approximated by the equation

$$
I_{21} / I_{20}=1+\left(g_{0}-1\right)\left\{1-\exp \left[-\left(t-t_{0}\right) / \tau\right]\right\} .
$$

Here, $I_{21}$ and $I_{20}$ are the intensities of beam 2 transmitted through the layer when beam 1 was switched on and off, respectively; $t_{0}$ is the time of switching beam 1 on; and $\tau$ is the response time or grating formation time constant. By approximation, it was found that the gain factor is $g_{0}=1.006$ and the response time is $\tau=$ $1.1 \mathrm{~s}$ in a field of $E_{0}=83.3 \mathrm{~V} / \mu \mathrm{m}$. An increase in applied field to $E_{0}=100 \mathrm{~V} / \mu \mathrm{m}$ leads to an increase in the gain factor to $g_{0}=1.017$ and a reduction in the response time to $\tau \approx 0.2 \mathrm{~s}$

The two-beam coupling gain coefficient $\Gamma$ was calculated by the conventional relationship [7]:

$$
\Gamma=\ln \left(\beta g_{0}\right) /\left(1+\beta-g_{0}\right) / L,
$$

where $\beta=I_{1}(0) / I_{2}(0)$ is the intensity ratio of the beams incident on the layer and $L$ is the optical path length of object beam 2 in the polymer layer.

Figure 5 illustrates the dependence of the twobeam coupling gain of beam 2 upon the applied field 
(a)

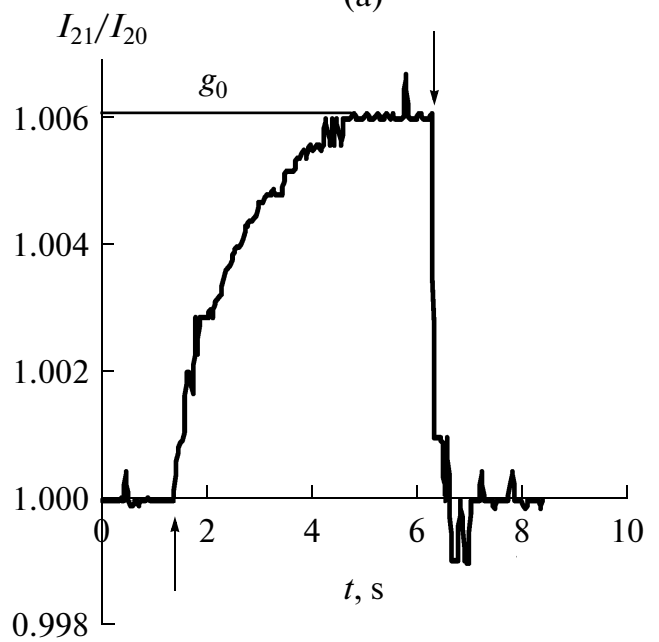

$I_{21} / I_{20}$

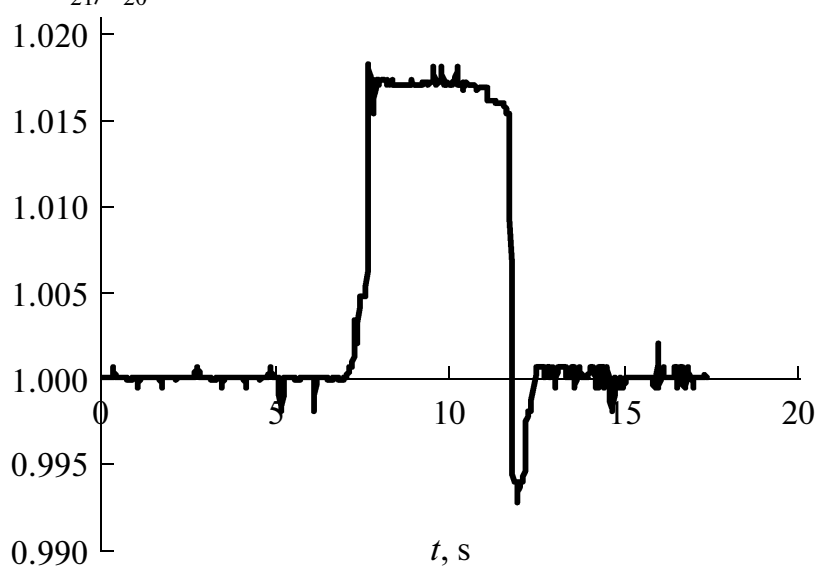

Fig. 4. Kinetic curves of amplification of beam 2 at $E_{0}=$ (a) 83.3 and (b) $100 \mathrm{~V} / \mu \mathrm{m}$. The upward and downward arrows at curve a mark the time of switching beam 1 on and off, respectively.

$E_{0}$ as measured at $I_{1}(0)=I_{2}(0)$. At $E_{0}=150 \mathrm{~V} / \mu \mathrm{m}$, the gain coefficient is $\Gamma=50 \mathrm{~cm}^{-1}$.

The dependence of the gain coefficient on the intensity ratio $\beta=I_{1}(0) / I_{2}(0)$ was also measured. The value of $\beta$ was increased by lowering the object-beam intensity $I_{2}(0)$. Figure 6 shows the pattern of this dependence as measured at $I_{1}(0)=0.072 \mathrm{~W} / \mathrm{cm}^{2}$ and $E_{0}=83.3 \mathrm{~V} / \mu \mathrm{m}$. There are an initial increase in the gain coefficient from 8 to $14 \mathrm{~cm}^{-1}$ with an increase in $\beta$ from 1 to 2.4 and its subsequent drop. This effect is of practical importance as it allows images to be corrected. Indeed, if a partially fogged image containing areas in which the intensity $I_{2}(0)$ decreases from

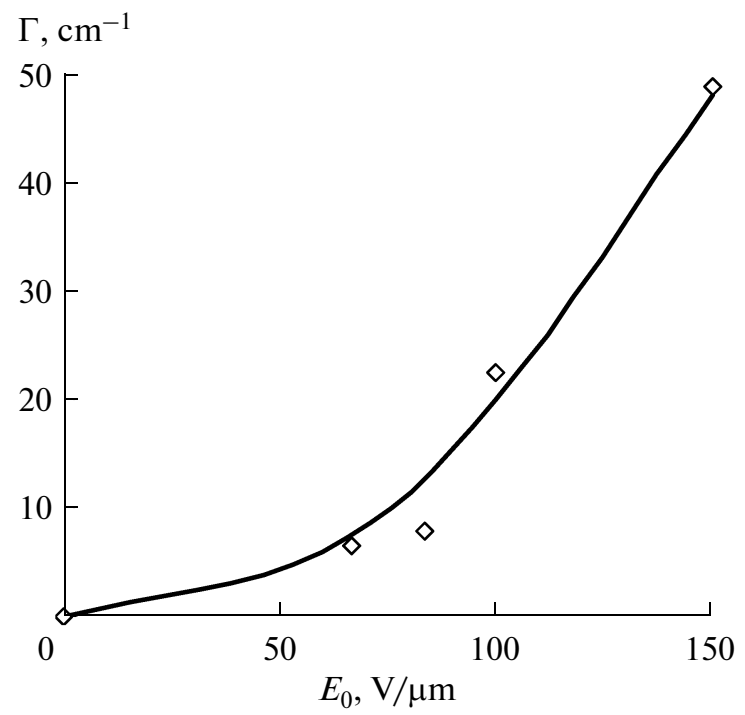

Fig. 5. Dependence of the two-beam coupling gain coefficient upon the applied constant field $E_{0}$.
$I_{2}(0)=I_{1}(0)$ to $I_{2}(0)=I_{1}(0) / a(a$ lies within $1-2.4)$, the fog effect weakens after passing through the PR layer, since the intensity of beam 2 is amplified in these regions according to the pattern of the curve in Fig. 6 .

\section{CONCLUSIONS}

The photoelectric and photorefractive characteristics of PVK composites with $0.15 \mathrm{wt} \%$ graphene at a wavelength of $532 \mathrm{~nm}$ have been measured.

The field dependence of the quantum efficiency for the generation of mobile charge carriers as determined from the dependence of photocurrent on the applied

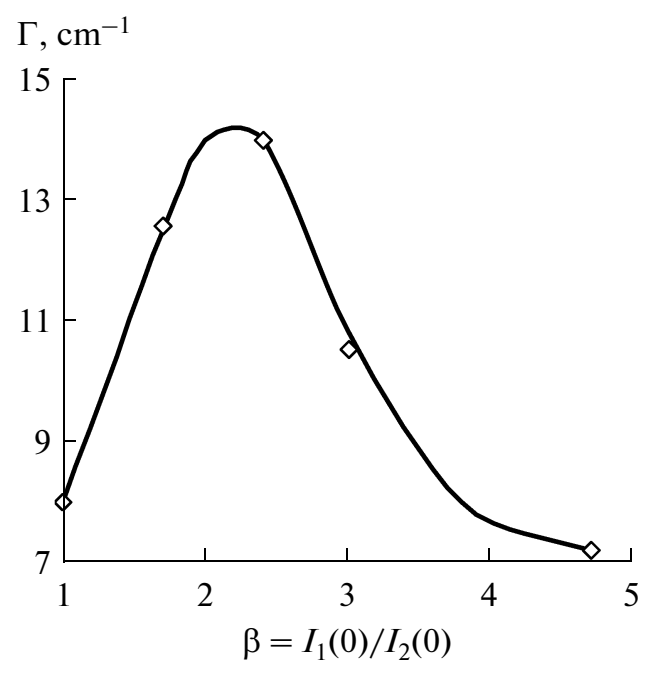

Fig. 6. Dependence of the two-beam coupling gain coefficient upon the intensity ratio of beams incident on the composite $I_{1}(0) / I_{2}(0)$ in a constant field of $E_{0}=$ $83.3 \mathrm{~V} / \mu \mathrm{m}$. 
electric field is well approximated by the Onsager equation calculated accurate to $E_{0}^{3}$ for the quantum yield of thermalized electron-hole pairs of $\varphi_{0}=1$ and their initial separation radius of $r_{0}=10.9 \AA$. This value greatly exceeds the separation radius of $r_{0}=9.8 \AA$ with $\varphi_{0}=1$ found at $1064 \mathrm{~nm}$ [1]. The long-wavelength edge of optical absorption by PVK lies near $365 \mathrm{~nm}$. Thus, the photogeneration of mobile carriers during illumination of the composite at $532 \mathrm{~nm}$ is due to photoexcitation of graphene as in the case of $1064 \mathrm{~nm}$. The measurement of the photorefractive properties has revealed that the two-beam coupling gain coefficient at equal intensities of input beams is $\Gamma=50 \mathrm{~cm}^{-1}$ at $E_{0}=150 \mathrm{~V} / \mu \mathrm{m}$. With $E_{0}=83.3 \mathrm{~V} / \mu \mathrm{m}$, it has been measured that the two-beam gain coefficient increases from 8 to $14 \mathrm{~cm}^{-1}$ as the intensity ratio of incident beams $I_{1}(0) / I_{2}(0)$ increases from 1 to 2.4 .

\section{ACKNOWLEDGMENTS}

This work was supported by the Russian Foundation for Basic Research, project no. 11-03-00260, and the Swedish Foundation for Strategic Research (SSF).

\section{REFERENCES}

1. Grishina, A.D., Krivenko, T.V., Savel'ev, V.V., Rychwalski, R.W., and Vannikov, A.V., High Energy Chem., 2013, vol. 47, no. 2, p. 46.

2. Vannikov, A.V. and Grishina, A.D., High Energy Chem., 2007, vol. 41, no. 3, p. 162.

3. Vannikov, A.V., Grishina, A.D., and Rychwalski, R.W., Carbon, 2011, vol. 49, no. 1, p. 311.

4. Vannikov, A.V., Gorbunova, Yu.G., Grishina, A.D., and Tsivadze, A.Yu., Prot. Met. Phys. Chem. Surf., 2013, vol. 49 , no. 1, p. 57.

5. Mozumder, A., J. Chem. Phys., 1974, vol. 60, no. 11, p. 4300 .

6. Akimov, I.A, Cherkasov, Yu.A, and Cherkashin, M.I., Sensibilizirovannyi fotoeffekt (Sensitized Photoelectric Effect), Moscow: Nauka, 1980.

7. Kukhtarev, N.V., Markov, V.B., Odulov, S.G., Soskin, M.S., and Vinetskii, V.L., Ferroelectrics, 1979, vol. 22, p. 949.

Translated by S. Zatonsky 